Revue musicale OICRM

\title{
Les paramètres de l'insertion professionnelle des musiciens au Québec. L'exemple de l'ensemble Magnitude6
}

\section{Marie-Pier Leduc}

Volume 1, numéro 1, 2012

Profession musicale au Québec

URI : https://id.erudit.org/iderudit/1055856ar

DOI : https://doi.org/10.7202/1055856ar

Aller au sommaire du numéro

Éditeur(s)

OICRM

ISSN

2368-7061 (numérique)

Découvrir la revue

Citer cet article

Leduc, M.-P. (2012). Les paramètres de l'insertion professionnelle des musiciens au Québec. L'exemple de l'ensemble Magnitude6. Revue musicale OICRM, 1(1), 20-35. https://doi.org/10.7202/1055856ar
Résumé de l'article

En raison du rôle partiel que joue la formation académique dans la gestion de l'incertitude liée à la profession d'interprète, l'analyse de l'insertion professionnelle des musiciens est des plus complexe. Cette recherche vise à comprendre comment s'opère le passage entre la formation académique et le marché professionnel, et plus particulièrement chez les musiciens jouant d'un cuivre. Ainsi, cette étude repose sur un travail de terrain réalisé auprès de l'ensemble montréalais Magnitude6, un quintette de cuivres avec batterie constitué d'étudiants en cours de professionnalisation et de professionnels établis. Cet article se présente en trois parties : la première dresse un portrait individuel des cinq interviewés destiné à faire ressortir les caractéristiques communes et distinctes de leur profil. La deuxième partie consiste en une analyse des stratégies de professionnalisation utilisées par les musiciens de Magnitude 6 basée sur la notion de gestion de l'incertitude. La dernière partie fera le point sur le rôle de la formation académique dans ces stratégies de professionnalisation. 


\title{
Les paramètres de l'insertion professionnelle des musiciens au Québec. L'exemple de l'ensemble Magnitude6
}

\author{
Marie-Pier Leduc
}

\begin{abstract}
Résumé
En raison du rôle partiel que joue la formation académique dans la gestion de l'incertitude liée à la profession d'interprète, l'analyse de l'insertion professionnelle des musiciens est des plus complexe. Cette recherche vise à comprendre comment s'opère le passage entre la formation académique et le marché professionnel, et plus particulièrement chez les musiciens jouant d'un cuivre. Ainsi, cette étude repose sur un travail de terrain réalisé auprès de l'ensemble montréalais Magnitude6, un quintette de cuivres avec batterie constitué d'étudiants en cours de professionnalisation et de professionnels établis. Cet article se présente en trois parties : la première dresse un portrait individuel des cinq interviewés destiné à faire ressortir les caractéristiques communes et distinctes de leur profil. La deuxième partie consiste en une analyse des stratégies de professionnalisation utilisées par les musiciens de Magnitude6 basée sur la notion de gestion de l'incertitude. La dernière partie fera le point sur le rôle de la formation académique dans ces stratégies de professionnalisation.

Mots clés : formation académique ; incertitude ; insertion professionnelle ; musiciens jouant d'un cuivre ; stratégies de professionnalisation.
\end{abstract}

\begin{abstract}
Because of the partial role of academic training in the management of the uncertainty associated with the profession of performer, the analysis of the professional integration of musicians is most complex. This research is intended to understand how the transition is made between the academic training and the professional market, especially among brass musicians. Thus, this study is based on a fieldwork conducted with the Montreal's Ensemble Magnitude6, a brass quintet with drums consisting of students being professionalized and of established professionals. This article has three parts: the first one gives an individual portrayal of the five respondents, intended to bring out the common and distinct features from their profile. The second part consists of an analysis of the professionalization strategies used by Magnitude6's musicians, based on the concept of uncertainty's management. The final part will review the role of academic training in these professionalization strategies.
\end{abstract}

Keywords: academic training; brass musicians; professional integration; professionalization strategies; uncertainty. 
Jusqu'à présent, peu de recherches ont porté sur l'insertion professionnelle des musiciens au Québec. Cet article vise à identifier un certain nombre de paramètres de la professionnalisation au Québec à partir de musiciens dont on entend généralement peu parler, soit ceux jouant d'un instrument associé à la famille des cuivres. C'est à partir de l'ensemble Magnitude6, un quintette de cuivres avec batterie, que nous nous proposons de travailler ${ }^{1}$. Puisqu'il s'agit d'une jeune formation constituée d'étudiants en cours de professionnalisation et de professionnels établis, Magnitude6, en tant que structure professionnelle, nous semble être un exemple intéressant pour étudier la trajectoire de musiciens jouant d'un cuivre et ainsi analyser le lien entre la formation académique et le marché professionnel. Cette recherche s'appuie sur l'hypothèse que la formation académique en interprétation, bien qu'étant quasi nécessaire pour atteindre un haut niveau instrumental, n'agit que partiellement sur l'accès au marché du travail.

Cette étude se développe en trois parties : la première dresse un portrait individuel des cinq interviewés destiné à faire ressortir les caractéristiques communes et distinctes de leur profil. La deuxième partie consiste en une analyse des stratégies de professionnalisation utilisées par les musiciens de Magnitude6 basée sur la notion de " modes de gestion de l'incertitude » mise de l'avant par Pierre-Michel Menger (2009, p. 203). La dernière partie fera le point sur le rôle de la formation académique dans ces stratégies de professionnalisation.

\section{PROFILS INDIVIDUELS}

Les cinq membres de l'ensemble Magnitude6 ont des profils présentant de nombreuses caractéristiques communes. Ils jouent tous d'un instrument appartenant à la famille des cuivres, ont tous une expérience à titre de pigiste ${ }^{2}$ sur la scène musicale montréalaise et ils ont tous reçu une formation musicale de haut niveau dans diverses institutions d'enseignement supérieur comme nous allons le voir plus loin. S'ils forment un groupe relativement homogène, il faut néanmoins tenir compte des particularités propres au profil de chacun des musiciens pour comprendre le rôle qu'ont joué les différentes stratégies de professionnalisation dans leur carrière respective. Cinq éléments clés ont été sélectionnés pour faire ressortir les similitudes et les singularités de leur profil : l'instrument joué, la formation académique, la carrière actuelle, les ambitions professionnelles ainsi que le rapport à la stabilité d'emploi qui est intimement lié à l'âge des musiciens.

\footnotetext{
1 À la base de cette recherche se trouvent des entretiens de nature semi-structurée réalisés entre le 31 mai 2011 et le 10 février 2012 avec cinq membres de l'ensemble Magnitude6, soit : Samuel LalandeMarkon, tuba ; Simon Jolicoeur-Côté, trombone ; Laurence Latreille-Gagné, cor ; Frédéric Demers, trompette ; et Thierry Champs, trompette (http://magnitude6.ca). Cette recherche a reçu l'aval des responsables de l'équipe de sociomusicologie du Laboratoire musique, histoire et société (LMHS) de l'Observatoire interdisciplinaire de création et de recherche en musique (OICRM), Michel Duchesneau et Danick Trottier.
}

2 Un musicien pigiste est un contractuel, bien souvent identifié sous l'étiquette de free-lance. 
Le producteur et le directeur artistique de Magnitude6, Samuel LalandeMarkon, est tubiste. Il a obtenu en 2008 un baccalauréat en interprétation de l'Université de Montréal et, en 2010, un Professional Studies Diploma du Mannes College à New York avec Alan Bear, le tubiste soliste du New York Philharmonic. Après ces deux années d'études à l'étranger, Lalande-Markon a entamé, à l'Université de Montréal, un doctorat en musicologie qu'il a ensuite délaissé pour entreprendre une formation en gestion d'organismes culturels à l'École des hautes études commerciales (HEC) de Montréal. En plus des fonctions qu'il occupe au sein de Magnitude6, il est actif comme tubiste pigiste et donne, à l'occasion, des cours de tuba.

Présentement dans la mi-vingtaine, Lalande-Markon ne cherche pas à obtenir, à court ou moyen terme, un poste comme tubiste dans un orchestre. Son idéal professionnel serait plutôt d'occuper un poste dans un domaine para-artistique, tel que la production d'évènements ou l'enseignement, lui donnant suffisamment de flexibilité pour prendre part à plusieurs projets en tant que travailleur autonome. Il recherche ainsi un équilibre entre une stabilité financière et un travail non routinier : "Mon idéal serait d'avoir un emploi contractuel deux à trois jours par semaine [...]. Tout ce que je veux pour les prochaines années, c'est d'avoir la flexibilité pour aller au bout de mes projets. »

Simon Jolicoeur-Côté, le tromboniste de Magnitude6, a effectué l'essentiel de sa formation académique avec Albert Devito. Il a complété avec lui un diplôme d'études collégiales au Cégep Saint-Laurent, un baccalauréat en interprétation à 1'Université de Montréal ainsi qu'une partie de sa maîtrise en interprétation. Jolicoeur-Côté est aussi tromboniste pour le groupe hip-hop Nul si découvert, professeur spécialiste ${ }^{3}$ de trombone dans une école primaire et secondaire, responsable de l'organisation liée à la présence de l'ensemble de cuivres aux collations des grades de l'Université de Montréal et est actif comme pigiste. De même que Lalande-Markon, Jolicoeur-Côté ne cherche pas, à court ou moyen terme, à obtenir un poste permanent dans un orchestre. Actuellement dans la mi-vingtaine, il préfère avant tout un travail non routinier.

À l'exception d'une année d'échange à Stuttgart, Laurence Latreille-Gagné, la corniste du quintette, a accompli l'ensemble de sa formation musicale au Conservatoire de musique de Montréal, où elle complète en ce moment sa maîtrise. En plus de son implication dans Magnitude6, Latreille-Gagné est professeure spécialiste de cor dans deux écoles secondaires et est active sur la scène musicale en tant que pigiste. Également dans la mi-vingtaine, elle cherche à obtenir un équilibre entre une activité non-routinière et une certaine stabilité financière. Pour ce faire, Latreille-Gagné souhaiterait occuper, à moyen terme, un poste dans un orchestre en parallèle à ses activités de pigiste.

L'ensemble Magnitude6 compte deux trompettistes, Frédéric Demers et Thierry Champs, qui sont tous les deux dans la mi-trentaine. Demers a effectué

3 Un professeur spécialiste est un professeur d'instrument qui donne des cours en privé ou en petit groupe à des élèves d'écoles primaires ou secondaires. 
plusieurs années d'études au Conservatoire de musique de Montréal où il a obtenu un Diplôme d'études supérieures II. Il a également complété une maîtrise en quintette de cuivres à 1'Université McGill et un doctorat en interprétation à 1'Université de Montréal. Demers est fort bien intégré au marché professionnel québécois. Il est très actif en tant que pigiste en plus d'être professeur de trompette au Conservatoire de musique de Val-d'Or et dans une école secondaire de la région de Montréal. Demers s'est, entre autres, fait connaître par la scène jeunesse, d'abord avec son quintette de cuivres Impact, puis par la mise sur pied du spectacle Les sept trompettes de Fred Piston dont il a donné plus de 350 représentations à travers le Québec.

Demers apprécie l'absence de routine associée à la carrière de musicien pigiste, mais tient également à la stabilité d'emploi qu'il a acquise à ce jour, notamment par son activité de professeur. À moyen terme, il cherche à développer sa carrière en tant que trompettiste soliste.

Thierry Champs, quant à lui, a un parcours académique qui diffère considérablement de celui des quatre autres interviewés. D'origine française, il a complété une grande partie de sa formation instrumentale dans son pays d'origine, soit au Conservatoire national de musique de Bayonne et au Conservatoire national de musique de Versailles. À son arrivée au Québec, il a suivi une formation en gestion financière informatisée, à la suite de quoi il a entrepris des études en enseignement de la musique à l'Université du Québec à Montréal (UQÀM) en parallèle à des études en interprétation au Conservatoire de musique de Montréal. Champs a également entamé un doctorat en interprétation à l'Université de Montréal. En plus d'être actif à titre de pigiste, il est trompettiste soliste à la Société de musique contemporaine du Québec (SMCQ), professeur de trompette en privé et a été professeur de musique dans une école secondaire. Il occupe, depuis peu, un poste de professeur de pédagogie musicale à l'UQÀM.

Une autre caractéristique propre au profil de Champs est l'importance qu'il accorde à la stabilité d'emploi. Celle-ci a toujours été importante pour lui, mais l'est encore plus depuis qu'il est père. Il considère avoir acquis un niveau d'insertion professionnelle et une stabilité d'emploi confortable, autant d'avantages professionnels qu'il souhaite conserver.

\section{LES STRATÉGIES DE PROFESSIONNALISATION}

Les travaux de Menger (2009, p. 203) ont démontré que la faible routinisation de l'exercice artistique a pour corollaire l'incertitude de la réussite de l'insertion professionnelle. Si le degré d'incertitude est réduit en France pour les interprètes classiques en raison de la forte institutionnalisation du milieu de la musique savante (voir Coulangeon 2008, p. 47), la réalité est bien différente au Québec. En effet, le marché québécois de la musique classique est beaucoup plus restreint ${ }^{4}$, et le diplôme universitaire, bien que presque toujours essentiel, ne garantit pas l'accès au marché du travail dans le domaine de la musique classique. 
Face à l'incertitude de leur insertion professionnelle, les musiciens de l'ensemble Magnitude6 ont développé des stratégies de professionnalisation diversifiées. Celles-ci peuvent être regroupées en trois modes de gestion de l'incertitude : 1) le recours à la pluriactivité et à la polyvalence ; 2) la création et l'entretien de réseaux professionnels; et 3) la mise sur pied et la participation à des structures professionnelles. Le recours à ces modes de gestion de l'incertitude s'effectue à des degrés variables par les membres de l'ensemble en fonction, par exemple, de leur instrument ${ }^{5}$, de leur formation, de leurs ambitions professionnelles, de leur âge et de leur rapport à la stabilité d'emploi. Les frontières qui délimitent ces trois modes de gestion de l'incertitude ne sont pas étanches. Au contraire, leur porosité permet à ces derniers de se nourrir mutuellement.

\section{La pluriactivité et la polyvalence}

Les musiciens de Magnitude6 ont tous recours à une ou plusieurs formes de diversification des activités pour gérer l'incertitude liée à leur insertion professionnelle. Trois formes de diversification des activités ont été identifiées dans le cadre de cette étude, s'inspirant de la définition proposée par Marie-Christine Bureau et Roberta Shapiro dans l'introduction du collectif L'artiste pluriel. Démultiplier l'activité pour vivre de son art (Bureau et Shapiro 2009, p. 19-20) ${ }^{6}$. La polyactivité réfère à 1'accumulation d'activités dans des champs différents, tandis que la pluriactivité désigne la pratique de plusieurs métiers dans un même champ. La polyvalence, quant à elle, renvoie à la capacité d'adaptabilité des musiciens à différents répertoires (classique, jazz, populaire, etc.) et à différents types d'ensembles (orchestre symphonique, orchestre de chambre, etc.).

À l'exception de Champs, qui a suivi une formation en gestion financière informatisée et qui a œuvré dans ce domaine durant six mois, les musiciens de Magnitude6 ne recourent pas à la polyactivité pour diversifier leurs activités. Ils font cependant tous appel à la pluriactivité et à la polyvalence.

La pluriactivité est vue par plusieurs membres de Magnitude6 comme un impératif pour vivre de la musique, une idée à laquelle certains membres ont été sensibilisés très tôt par des acteurs du milieu musical. Par exemple, un enseignant a déclaré à Jolicoeur-Côté qu'il fallait, pour vivre de la musique, pratiquer deux des trois activités que sont l'interprétation, l'enseignement et l'arrangement : « Il y a un professeur à l'université qui disait qu'il y a trois choses dans la vie : jouer, arranger et enseigner. Si tu ne fais pas deux de ces trois choses-là, tu ne pourras pas gagner ta vie en musique. » À ces trois occupations s'ajoutent d'autres activités de diversification possibles telles

$5 \quad$ Il est, par exemple, plus facile pour un trompettiste qu'un tubiste de jouer régulièrement.

6 Les définitions de deux des trois formes de diversification des activités proposées par Bureau et Shapiro, soit celles de la polyactivité et de la pluriactivité, ont été reprises telles quelles, tandis que la définition de la polyvalence a été adaptée pour correspondre davantage à la réalité des musiciens de l'ensemble Magnitude6. Ainsi, dans la présente étude, la polyvalence ne correspond pas à « l'exercice de plusieurs métiers au sein d'un même collectif de travail », mais à la pratique de plusieurs répertoires au sein de différents ensembles. 
que l'enregistrement, la composition et la production d'évènements. Cependant, la combinaison de l'interprétation à l'enseignement est la forme la plus fréquente de diversification des activités chez les musiciens de Magnitude6.

C'est le cas de Jolicoeur-Côté qui se définit avant tout comme interprète, mais qui est aussi professeur spécialiste de trombone dans une école primaire et secondaire. Quant à Latreille-Gagné, elle enseigne le cor à titre de professeure spécialiste dans deux écoles secondaires, un emploi qui lui offre une grande flexibilité puisqu'elle peut facilement s'y faire remplacer lorsque survient un conflit d'horaire avec un contrat d'interprète, activité qu'elle va toujours prioriser par rapport à l'enseignement.

L'enseignement intéresse aussi Lalande-Markon dont l'idéal professionnel serait d'occuper un emploi à mi-temps dans un domaine para-musical, lui laissant ainsi assez de temps pour s'activer à titre de travailleur autonome. C'est en ayant en tête l'enseignement de matières théoriques au cégep que Lalande-Markon a entamé un doctorat en musicologie, qu'il a ensuite délaissé pour entreprendre un diplôme en gestion d'organismes culturels à HEC de Montréal. Ce diplôme a pour but de l'outiller pour devenir producteur d'évènements musicaux, rôle qu'il joue déjà au sein de Magnitude6, en plus d'être tubiste pigiste et d'avoir quelques élèves en enseignement privé. Pour Lalande-Markon, il est important de posséder plusieurs « cartes » dans son jeu, pour reprendre son expression.

L'enseignement joue également un très grand rôle dans la carrière des deux trompettistes de Magnitude6. Bien que les revenus de Demers proviennent davantage de l'enseignement, il se définit autant comme interprète que professeur en raison du temps qu'il investit dans ses projets d'interprétation. La non-routinisation de la carrière $d$ 'instrumentiste est très appréciée par Demers, mais, en raison de ses besoins de stabilité financière qui évoluent avec l'âge, ce dernier laisse une place de plus en plus grande à l'enseignement, ce qui lui assure une sécurité financière. Il balise ainsi les risques liés à sa carrière d'interprète.

\section{Exemple audio 1 : Frédéric Demers. É Ecouter.}

Champs, pour qui la stabilité d'emploi est très importante, a orienté ses choix de carrière à la fois vers l'interprétation et l'enseignement de la musique (enseignement de la trompette en privé, enseignement de la musique au secondaire, mise sur pied d'un camp musical, etc.). Bien qu'il soit encore très actif comme interprète sur la scène montréalaise, il se considère avant tout comme un enseignant, surtout depuis qu'il a obtenu un poste de professeur en pédagogie musicale à l'UQÀM. Cette stabilité d'emploi lui permet une liberté dans le choix de ses projets en tant qu'interprète. Ainsi, il peut se concentrer sur des projets qui ne sont pas nécessairement financièrement très lucratifs, mais qu'il juge artistiquement et humainement enrichissants tels que Magnitude6.

À la lumière de ces témoignages, force est de constater que l'organisation de la pluriactivité, ou de la " composition du portefeuille » selon l'expression utilisée par Menger, se transforme en fonction de la progression de la carrière. Chez les plus jeunes musiciens de Magnitude6, l'organisation des activités correspond à une dispersion assez aléatoire des activités, mais est conduite par le souci d'une stabilité financière à moyen terme afin d'assurer une continuité de leur activité instrumentale. 
En revanche, chez les deux trompettistes, qui sont plus intégrés au marché professionnel, la situation qui prévaut est davantage concentrée autour de pôles dominants (voir Menger 2009, p. 218-219).

La polyvalence, contrairement à la pluriactivité, prend un sens très différent selon l'instrument joué. Ainsi, la diversité de répertoire associée au cor est beaucoup plus restreinte que celle associée à la trompette. Pour Latreille-Gagné, la polyvalence renvoie davantage à sa capacité d'adaptabilité à différentes formations instrumentales qu'à différents répertoires, bien qu'être à l'aise avec le répertoire classique et contemporain ainsi que de jouer du cor naturel peut se traduire comme un avantage. LalandeMarkon se retrouve dans une situation similaire avec le tuba comme instrument. Pour lui, la polyvalence correspond à la faculté d'adaptation à différents types de formations instrumentales, ainsi qu'à la capacité de jouer des différents tubas ténors, basses et contrebasses. Les possibilités de diversification du répertoire sont plus grandes pour le trombone qui peut être joué tant dans des ensembles classiques, jazz et populaires.

Jolicoeur-Côté, qui est lui-même actif dans un ensemble populaire, est d'avis qu'un tromboniste habile dans plusieurs répertoires est susceptible d'être plus souvent engagé : « Je crois qu'un tromboniste qui joue du classique, du contemporain et du jazz va se faire plus souvent appeler. " Il a d'ailleurs acquis des aptitudes en jazz avec son groupe de musique populaire. Il ne cherche cependant pas à acquérir plus d'habiletés dans ce répertoire, car il souhaite avant tout se concentrer au développement d'un son classique ${ }^{7}$. La polyvalence prend donc, pour Jolicoeur-Côté, un sens comparable à celui défini pour Latreille-Gagné et Lalande-Markon, et s'articule autour de l'adaptabilité à différentes formations instrumentales et de la capacité de jouer de plusieurs sortes de trombones, principalement le ténor et l'alto dans son cas.

La capacité d'adaptabilité à différents répertoires est un mode de gestion de l'incertitude qui s'applique tout particulièrement aux deux trompettistes de Magnitude6. Très conscients des possibilités de polyvalence qu'offre leur instrument, Demers et Champs ont exploité avec succès cette dimension dans leur insertion professionnelle. La polyvalence serait même une qualité presque indispensable pour les trompettistes pigistes au Québec, et, selon Champs, le manque de polyvalence chez certains trompettistes pourrait expliquer qu'ils doivent ultimement quitter la profession.

\section{Exemple audio 2: Thierry Champs. Écouter.}

En raison de la petite taille du marché québécois, Demers considère qu'il n'est pas possible de vivre d'une spécialité comme celle de la trompette baroque en tant que trompettiste pigiste, et affirme que le fait d'être capable de jouer autant du répertoire classique, jazz, populaire, contemporain et baroque lui a beaucoup servi : "Au Québec, c'est tout simplement un incontournable. Si on était en Europe, et puis que j'étais spécialisé en trompette baroque, il y aurait un moyen de vivre que de ça. [...] Au Québec, la polyvalence m’a énormément aidé. » 
Le recours à la pluriactivité et à la polyvalence peut être comparé, comme l'a fait Menger (2009, p. 216), à une forme de diversification d'un portefeuille de placements afin de réduire le risque de l'investissement personnel. Cependant, ces deux formes de diversification des activités ne peuvent être limitées à un mode de gestion de l'incertitude. La pluriactivité, et plus spécifiquement l'enseignement, est vécue par plusieurs musiciens de Magnitude6 comme une forme de partage et un don de soi donnant un sens au métier d'interprète. Les musiciens de l'ensemble continueraient à exercer l'enseignement même si cela n'était plus une nécessité financière.

Exemple audio 3 : Simon Jolicoeur-Côté. Écouter.

Aussi, la polyvalence de répertoire n'est pas uniquement considérée par les trompettistes comme une façon de jouer plus souvent. Pour Demers et Champs, l'apprentissage du jazz est venu nourrir leur jeu instrumental classique, notamment en les aidant à adopter une attitude plus décontractée face à l'interprétation.

En résumé, il semble beaucoup plus avantageux pour de jeunes professionnels d'avoir recours à la pluriactivité qu'à la polyactivité puisque la fréquentation du milieu musical permet la création de nouveaux réseaux de contacts, le deuxième mode de gestion de l'incertitude utilisé par les musiciens de Magnitude6.

\section{La création et l'entretien de réseaux professionnels}

La création et l'entretien de réseaux professionnels sont les modes de gestion de l'incertitude ayant été le plus explicitement exprimés par les musiciens de Magnitude6 en tant que stratégie de professionnalisation. Tous sont conscients de l'importance du capital social, dans le sens d'" ensemble des ressources actuelles ou potentielles qui sont liées à la possession d'un réseau durable de relations plus ou moins institutionnalisées d'interconnaissance et d'interreconnaissance " (Bourdieu 1980, p. 2), pour accéder au marché du travail.

Inspirée de la définition de la notion de réseau mise de l'avant par Françoise Liot (2004, p. 76), l'idée de réseau telle qu'elle est utilisée ici tiendra compte non seulement des conventions et des valeurs régissant le monde de l'art dans lequel évoluent les musiciens de Magnitude6, mais également des relations entre les différents types d'acteurs qui véhiculent ces "règles non écrites ». En ce sens, les réseaux dont il est question ici se rattachent à la notion de monde de l'art de Howard S. Becker ([1988]2010, p. 88) : les processus sociaux, le partage des conventions et les actions communes derrière les mondes de l'art se trouvent stabilisés par ces réseaux. Le fait d'entretenir un réseau diversifié et formé de relations durables permet d'assurer aux musiciens un équilibre entre la variété des projets et une certaine stabilité d'emploi (voir Roux 2011, p. 12).

Conscients de l'importance des réseaux pour leur professionnalisation, les musiciens de Magnitude6 cherchent à fréquenter divers lieux de socialisation, tels que les salles de concert et les institutions d'enseignement. Ainsi, la fréquentation de salles de concert est, pour Latreille-Gagné, plus qu'un simple souci, une préoccupation presque constante comparable à celle de la pratique instrumentale : "Toutes les actions sont importantes. Par exemple, si je vais à un concert, je peux me faire des amis qui vont me faire avoir des contrats. Tout a une incidence. » 
En plus de créer des relations, il est important de les entretenir. À son retour de New York, où il a étudié deux ans, Lalande-Markon a constaté que son intégration à la vie professionnelle sur la scène montréalaise était légèrement plus ardue qu'avant son départ en raison de l'effritement du réseau qu'il n'a pu entretenir, une situation qui s'est résorbée après une année d'activité. Les avantages d'aller étudier à l'étranger s'accompagnent du désavantage de la perte de visibilité dans son milieu d'origine, un revers de la médaille dont Latreille-Gagné est bien consciente : «Le fait que j'ai étudié à l'étranger rend les gens curieux, mais en même temps, ils n'ont pas entendu parler de moi pendant un an. "

La fréquentation des lieux de socialisation permet la rencontre de personnes pivots, c'est-à-dire des acteurs du milieu musical possédant un fort capital social et jouant un rôle de pont, permettant ainsi aux étudiants et aux jeunes professionnels d'intégrer un réseau plus vaste. Dans le réseau des membres de Magnitude6, les relations avec ces personnes pivots se construisent généralement à partir d'affinités électives. Pour plusieurs des musiciens de l'ensemble, c'est leur professeur d'instrument qui a joué ce rôle décisif dans leur insertion professionnelle. À cet effet, l'exemple de Jolicoeur-Côté est particulièrement révélateur puisqu'Albert Devito, qui a été son professeur pendant 11 ans, lui a donné accès à de nombreux contrats d'engagement et, surtout, lui a confié la responsabilité de s'occuper de l'organisation reliée à la présence de l'ensemble de cuivres aux collations des grades de l'Université de Montréal.

Il y a aussi l'exemple de Demers, dont la professeure de trompette au Conservatoire de musique de Montréal, Manon Lafrance, lui a permis d'entrer sur les listes des surnuméraires de certaines grandes formations.

\section{Exemple audio 4 : Frédéric Demers. Écouter.}

On observe un phénomène similaire chez Latreille-Gagné : "Je sais que les orchestres professionnels qui m'appellent, c'est parce qu'une fois mon professeur au conservatoire m'a référée pour un concert. À partir de ce moment-là, j'ai eu mon nom sur la liste, et si je joue bien, ils vont me rappeler [...]. »

$\mathrm{Au}$ sein même de Magnitude6, certains membres jouent le rôle de pivot. C'est notamment le cas de Demers qui, de par sa forte intégration professionnelle ainsi qu'en raison des nombreux projets auxquels il avait déjà pris part, a pu introduire l'ensemble et plus particulièrement Lalande-Markon à un réseau avec lequel il s'était déjà familiarisé.

\section{Exemple audio 5 : Frédéric Demers. Écouter.}

En plus de permettre la création de réseaux de contacts, la fréquentation de lieux de socialisation permet aux étudiants et aux jeunes professionnels de se familiariser avec les " codes langagiers et procéduriers mis en œuvre dans le milieu de l'art " (Liot 2004, p. 180). Puisque les réseaux de contacts se basent sur une affinité élective, il est essentiel pour les musiciens d'être au fait des valeurs et des conventions en usage dans leur milieu professionnel. Pour les membres de Magnitude6, les valeurs s'organisent autour de trois pôles : les qualités humaines, les qualités professionnelles et les qualités techniques.

Pour œuvrer sur la scène montréalaise en tant que pigiste, il est important de posséder un bon niveau instrumental, mais cela est loin d'être le seul critère nécessaire 
pour être engagé. En fait, puisque les réseaux de contacts professionnels sont souvent les mêmes que les réseaux d'amitié, les qualités humaines et professionnelles sont considérées par les membres de Magnitude6 comme aussi sinon plus importantes que les capacités instrumentales.

\section{Exemple audio 6 : Laurence Latreille-Gagné. Écouter.}

Il y a un consensus chez les musiciens de l'ensemble à l'effet que les réseaux basés sur la camaraderie sincère sont les meilleurs puisque les plus durables.

\section{Exemple audio 7 : Samuel Lalande-Markon. Écouter.}

La relation entre Demers et Champs en est un très bon exemple puisque leur amitié les a emmenés à être solidaires dans le partage des engagements émanant du milieu professionnel.

\section{Exemple audio 8: Thierry Champs. Écouter.}

Dans les domaines pour lesquels les diplômes n'ont qu'une valeur secondaire par rapport à l'expérience, la réputation joue un rôle primordial dans l'accès au travail. Comprise ainsi comme un processus social, la réputation rejoint l'analyse proposée par Becker ([1988]2010, p. 349) dans Les mondes de l'art : elle seconde la valeur qui est accordée à un artiste. Dans le monde de l'art au sein duquel les musiciens de Magnitude6 évoluent, la réputation est basée sur le système de valeurs dont il a été question précédemment. Ainsi, le fait de participer et de gagner des concours, bien que pouvant être utile, n'est pas une nécessité pour être actif à titre de pigiste sur la scène montréalaise, alors que, selon Jolicoeur-Côté, la ponctualité, la disponibilité, l'humilité et l'adaptabilité sont parmi les qualités les plus importantes à posséder pour avoir une bonne réputation.

Aussi, le fait d'être réputé comme ayant de nombreux engagements à son actif agit à titre de garantie de l'employabilité des musiciens. L'âge et l'expérience des instrumentistes sont donc des facteurs importants dans l'accès au marché du travail puisque, tel qu'en témoigne Jolicoeur-Côté, l'emploi engendre de l'emploi.

\section{Exemple audio 9 : Simon Jolicoeur-Côté. Écouter.}

Comme le souligne Liot : "La professionnalisation est soumise aux capacités d'un individu à mobiliser des ressources acquises au cours d'une vie relationnelle » (Liot 2004, p. 182). Un certain capital social a été acquis par les membres de Magnitude6 en fréquentant divers lieux de socialisation, tels que les établissements d'enseignement et les salles de concert. Ces lieux sont également l'endroit où ils ont été sensibilisés à un bon nombre de conventions du milieu musical et plus spécifiquement de l'environnement constitué par les musiciens qui jouent des cuivres. Cependant, un certain nombre de ces conventions ne peuvent être assimilées exclusivement en côtoyant un cercle social, mais sont susceptibles d'être intégrées uniquement par l'expérience concrète. Le troisième mode de gestion de l'incertitude utilisé par les musiciens de Magnitude6, soit la mise sur pied et la participation à des structures professionnelles, permet cet apprentissage sur le tas si essentiel à la professionnalisation. 
Les frontières entre le mode de gestion de l'incertitude que sont la création et l'entretien de réseaux et celui de la mise sur pied et de la participation à des structures professionnelles sont particulièrement poreuses. En fait, puisque les structures professionnelles sont un lieu de socialisation, cette troisième stratégie de professionnalisation peut être vue comme découlant de la deuxième. Mais, en raison des bénéfices associés à l'implication dans diverses structures professionnelles qui transcendent ceux associés au réseautage, cette dernière stratégie est considérée comme un mode de gestion de l'incertitude à part entière. En effet, la mise sur pied et la participation à des structures professionnelles donnent l'occasion aux étudiants et aux jeunes professionnels d'acquérir une expérience professionnelle et permettent un transfert de crédibilité et de compétences de l'ensemble vers ses membres et vice-versa. Les structures professionnelles jouent également le rôle de pont entre la fin de leur cheminement académique et leur pleine intégration professionnelle.

Les structures professionnelles dans lesquelles s'impliquent les musiciens de Magnitude6 sont très diverses, passant de l'orchestre symphonique à l'ensemble de chambre et incluant les carrières individuelles des interprètes lorsque ceux-ci cherchent à mettre sur pied des projets dans le cadre de leur carrière de soliste. Les simulations de structures professionnelles telles que les ensembles formés au sein des institutions d'enseignement ainsi que les structures semi-professionnelles ${ }^{8}$, sont incluses dans ce troisième mode de gestion de l'incertitude, puisque ces formations sont basées sur un modèle similaire à celui des ensembles professionnels et offrent des avantages dans l'insertion professionnelle à ceux qui y participent.

Tous les musiciens de l'ensemble ont insisté sur l'importance de participer au plus grand nombre possible de projets pour amorcer une carrière, puisqu'ils offrent une visibilité à l'interprète, lui donnent l'occasion de créer de nouveaux réseaux et de restreindre une certaine compétition. La recherche d'ubiquité est posée en utopie puisqu'ils visent à être " partout à la fois ", pour reprendre les mots de Latreille-Gagné. Ainsi, la participation à des projets de façon bénévole peut être, à long terme, très profitable, comme l'ont remarqué Jolicoeur-Côté et Champs.

\section{Exemple audio 10 : Simon Jolicoeur-Côté. Écouter.}

La participation à ces ensembles permet également l'apprentissage de conventions qui ne peuvent être acquises que par l'expérience. En d'autres mots, la collaboration à diverses structures professionnelles et semi-professionnelles permet l'accumulation d'expériences professionnelles.

L'ensemble de cuivres Chœur de métal $^{9}$ semble avoir été, pour quelques années, un catalyseur pour la professionnalisation des cuivres à Montréal. Cet ensemble a,

8 Les structures semi-professionnelles telles que les orchestres de quartier sont généralement composées de musiciens en cours de formation, d'anciens musiciens qui se sont réorientés vers une autre carrière et de quelques professionnels qui sont souvent les chefs de section.

9 Chœur de métal est un ensemble de cuivres et percussions de 18 musiciens (www.choeurdemetal.com). 
entre autres, été profitable pour Lalande-Markon qui, à la suite de sa collaboration avec Chœur de métal, a eu plusieurs autres occasions de jouer.

Exemple audio 11 : Samuel Lalande-Markon. Écouter.

D'ailleurs, c'est à la suite d'une participation à un concert de Chœur de métal que Latreille-Gagné a intégré Magnitude6, et son implication dans ce dernier a consécutivement débouché sur d'autres contrats dans divers ensembles.

Les structures professionnelles sont également le théâtre d'un transfert de crédibilité. Le fait d'avoir joué avec un ensemble réputé donne une certaine crédibilité à l'interprète. Dans une formation de taille plus modeste comme Magnitude6, l'ensemble apporte une crédibilité à ses membres, mais les membres eux-mêmes confèrent un certain capital au groupe. Ce transfert de crédibilité ne se fait pas de façon uniforme pour chacun des membres, en raison de leur réputation inégale. C'est ainsi que la réputation de Demers et de Champs rejaillit favorablement sur Magnitude6 et crédibilise l'ensemble de ses musiciens, comme en témoigne Jolicoeur-Côté : "Lorsque tu dis qu'il y a Frédéric Demers et Thierry Champs dans le quintette, ça donne une crédibilité à l'ensemble. L'ensemble nous apporte une crédibilité et on apporte une crédibilité à l'ensemble. »

Face à un milieu où très peu de postes s'ouvrent dans les orchestres permanents, et le nombre réduit d'engagements à la pige disponibles, les jeunes musiciens se doivent d'être entreprenants et créatifs. Si cette situation est valable pour tous les étudiants et les jeunes professionnels qui jouent d'un cuivre, elle l'était particulièrement pour Lalande-Markon au moment de terminer son baccalauréat. En fondant Magnitude6, il s'assurait de rester dans le milieu de l'interprétation en attendant de se créer une place dans le marché de l'emploi alors particulièrement saturé pour les tubistes au Québec. Il s'est « tenu en forme », pour utiliser ses mots. Bien entendu, Magnitude6 ne se limite pas à un ensemble mis sur pied « en attendant d'avoir mieux ». En plus d'être un moyen de rester actif sur la scène montréalaise, l'ensemble est considéré comme artistiquement et humainement stimulant par tous ses musiciens, et c'est pourquoi il peut aspirer à durer.

Une situation comparable à celle de Lalande-Markon a été vécue par Demers à la suite de l'obtention de son doctorat en interprétation en 2006. Devant une situation difficile, celle de ne pas pouvoir vivre de la pratique de la trompette, il met sur pied un spectacle jeunesse, Les sept trompettes de Fred Piston. Avec plus de 350 représentations à ce jour, le projet lui a permis de s'assurer une stabilité financière le temps que son intégration professionnelle soit plus assurée. Affilié aux Jeunesses musicales du Canada, Demers a pu éviter les lourdes tâches administratives entourant la production de ce spectacle, lui laissant ainsi du temps pour promouvoir sa carrière de trompettiste soliste et pour être actif dans le milieu de la pige.

\section{Exemple audio 12 : Frédéric Demers. Écouter.}

La mise sur pied et la participation à des structures professionnelles jouent donc un rôle considérable dans l'insertion professionnelle des instrumentistes. Pour les jeunes membres de l'ensemble, Magnitude6 est non seulement un projet stimulant, mais également un tremplin vers une intégration professionnelle plus complète. 
Pour Champs et Demers, qui sont déjà très bien intégrés en tant que pigistes sur la scène montréalaise, prendre part à Magnitude6 ne fait pas partie d'un mode de gestion de l'incertitude comme cela est le cas pour Lalande-Markon, Jolicoeur-Côté et Latreille-Gagné ; c'est plutôt la nature du projet artistique ainsi que l'atmosphère au sein de la formation qui les a motivés à s'engager dans l'ensemble.

\section{LE RÔLE DE LA FORMATION DANS LA GESTION DE L'INCERTITUDE}

Puisque les stratégies de professionnalisation des membres de Magnitude6 sont regroupées autour des trois modes de gestions de l'incertitude dont il est question ici, le rôle des formations collégiale et universitaire des musiciens a été, jusqu'à présent, très peu abordé. Bien que n'étant pas un mode de gestion de l'incertitude en soi, le parcours académique peut jouer un rôle très important dans l'insertion professionnelle des musiciens puisque, comme l'indique Menger :

L'une des fonctions de la fréquentation des établissements supérieurs d'enseignement artistique est [...] de familiariser l'apprenti artiste avec les milieux artistiques dans lesquels il a l'espoir de s'insérer, et de lui procurer un certain nombre d'expériences de travail avant même d'achever ses études (Menger 2009,p. 2148).

Cette fonction est remplie inégalement par les différentes institutions académiques, voire au sein d'un même établissement d'enseignement. Cela est dû, entre autres, à une caractéristique inhérente à l'apprentissage de la musique : les cours d'instrument en privé avec des professeurs possédant chacun un niveau de capital social très variable.

Le cheminement académique agit comme une variable pouvant intervenir favorablement sur ces trois modes de gestion de l'incertitude, offrant ainsi un " avantage cumulatif » (ibid., p. 328) à ses diplômés. Cependant, si ces avantages deviennent la norme dans les institutions d'enseignement, l'étudiant qui, pour diverses raisons, ne peut en profiter sera désavantagé. Raymonde Moulin précise, dans le cas des arts visuels, que

l'efficacité de l'école se mesure à l'opportunité qu'elle offre aux élèves de pénétrer dans le monde de l'art et dans le marché, en même temps qu'à l'aptitude développée chez eux de s'y reconnaître et de s'y faire connaître. [...] L'école, en même temps qu'elle est un lieu où se constituent les réseaux d'interconnaissance, initie les élèves à la topographie de l'espace artistique et aux stratégies imposées par le marché (Moulin 1992, p. 316).

Les divers parcours académiques suivis par les membres de Magnitude6 semblent leur avoir donné que très peu d'opportunités pour diversifier leurs activités, et même, dans certain cas, aucune. Si des cours de techniques d'enseignement en privé, d'enregistrement en studio et d'arrangement sont dispensés par certaines institutions universitaires dans le cadre du cursus en interprétation, l'offre de cours outillant les interprètes relativement à la pluriactivité reste restreinte. Qui plus est, elle est souvent méconnue des aspirants bacheliers qui, d'ailleurs, ne sont souvent pas encore 
conscients de tous les aspects de la réalité du métier de musicien et ne tendent pas à suivre de tels cours ${ }^{10}$.

En fait, le besoin de s'outiller au sujet de la pluriactivité semble lié au rapport des musiciens à la stabilité d'emploi, qui, de façon générale, évolue avec l'âge et tend à devenir un paramètre déterminant une fois que le marché du travail devient réalité. C'est donc souvent avec le recul que les interprètes ressentent le besoin de posséder des outils relatifs à la pluriactivité. Champs, pour qui la stabilité d'emploi a toujours été importante, s'est tout simplement doté d'un diplôme certifiant ses capacités à enseigner la musique en plus de ses diplômes en interprétation.

Aussi, la sensibilisation aux avantages de la polyvalence dépend souvent du professeur d'instrument et de sa propre sensibilité à ce sujet. De manière générale, la formation des musiciens de Magnitude6 a été presque exclusivement orientée vers le répertoire classique. Le développement de la capacité d'adaptabilité aux différents répertoires est alors laissé à la curiosité des étudiants qui peuvent, par exemple, participer de façon extrascolaire à des ensembles pratiquant divers styles.

Pour les membres de Magnitude6, les institutions d'enseignement ont joué un rôle beaucoup plus important dans la création et l'entretien de réseaux que dans la diversification des activités. Les écoles et les universités semblent être des lieux de socialisation particulièrement profitables, à un point tel que Jolicoeur-Côté envisage même d'entreprendre un autre diplôme après sa maîtrise uniquement pour créer de nouveaux réseaux de contacts.

La fréquentation de ces lieux de socialisation a été bénéfique pour tous les musiciens de l'ensemble, mais de façon très inégale. En effet, au sein d'une même institution, les étudiants n'auront pas tous la chance de fréquenter les mêmes personnes pivots, puisque celles-ci sont souvent un professeur privé d'instrument. Par exemple, Lalande-Markon et Jolicoeur-Côté, qui ont fait leur baccalauréat dans la même institution durant les mêmes années, y ont fait des expériences de réseautage très différentes. Pour le premier, les réseaux de contacts se sont principalement créés par le biais d'engagements professionnels, tandis que pour le second, les réseaux de contacts ont été en grande majorité créés dans le cadre de son parcours académique.

Les universités et les conservatoires offrent généralement plusieurs opportunités de participation à des structures de nature professionnalisante comme les orchestres universitaires. En s'impliquant dans ceux-ci, les étudiants gagnent en visibilité, ce qui, selon Latreille-Gagné, est essentiel pour œuvrer en tant que pigiste. La participation à ces formations permet également aux jeunes interprètes de se familiariser avec les conventions en vigueur dans les grands et petits ensembles. Cependant, les institutions d'enseignement offrent très peu d'occasions aux étudiants d'apprendre à gérer une carrière de pigiste ou de soliste, ce que Demers interprète comme un manque sérieux à la formation.

10 C'est le cas, entre autres, du cours «Le métier de musicien » dispensé par la Faculté de musique de 1'Université de Montréal dont très peu de musiciens de Magnitude6 ont entendu parler. 
Comme il fut mentionné plus haut, bien des jeunes professionnels se voient obligés de mettre sur pied leur propre structure professionnelle afin d'avoir la possibilité de jouer. Plusieurs musiciens de Magnitude6 considèrent ne pas avoir suffisamment eu la chance, durant leur parcours académique, de s'outiller pour faire face aux diverses réalités de la vie de musiciens pigistes comme la mise sur pied d'une structure professionnelle qui se révèle être une lourde tâche de gestion, ce dont témoigne Lalande-Markon.

\section{Exemple audio 13 : Samuel Lalande-Markon. Écouter.}

Demers affirme même qu'il peut souvent passer autant de temps, dans une journée, à s'occuper de tâches administratives qu'à jouer, d'où l'importance, selon lui, d'avoir des connaissances dans ce domaine. Sur ce troisième mode de gestion de l'incertitude, les institutions d'enseignement jouent un rôle mitigé. Il reste à savoir toutefois si des outils comme des cours portant sur la mise sur pied de structures professionnelles rencontreraient un accueil favorable de la part des étudiants de musique au moment où la connaissance de l'instrument a préséance sur l'acquisition des autres formes de connaissance liées à la musique. En d'autres mots, comme réponse à l'incertitude, cet outillage devient pertinent pour ces jeunes professionnels une fois qu'ils confrontent le marché du travail.

Les institutions d'enseignement agissent donc de façon inégale sur les trois modes de gestion de l'incertitude précédemment cités. La formation académique suivie par les membres de Magnitude6 paraît incomplète puisqu'elle semble être en grande partie orientée vers la formation de musicien d'orchestres. Cependant, obtenir un poste permanent dans un orchestre est une situation professionnelle à laquelle les cinq interviewés ne peuvent aspirer qu'à moyen ou à long terme et sans assurance que cela se réalise véritablement. Cette option est par conséquent exclue des ambitions professionnelles d'au moins trois d'entre eux. Toutefois, les institutions d'enseignement semblent avoir agi positivement sur leur insertion professionnelle de par les occasions d'acquérir une visibilité et de créer des réseaux de contacts.

\section{CONCLUSION}

L'étude des paramètres d'insertion professionnelle à partir de l'exemple de Mangitude6 fait apparaître l'utilisation de stratégies de professionnalisation organisées autour de trois modes de gestion de l'incertitude : le recours à la pluriactivité et à la polyvalence, la création et l'entretien de réseaux, ainsi que la mise sur pied et la participation à des structures professionnelles. Les témoignages des cinq interviewés révèlent que leur cheminement académique, en n'intervenant que partiellement dans ces stratégies de professionnalisation, agit comme un agent incomplet dans leur insertion professionnelle. Il ne faut cependant pas négliger le rôle fondamental qu'a joué leur formation universitaire dans l'acquisition d'un niveau élevé de compétences instrumentales nécessaire pour accéder au marché professionnel.

La formation en interprétation qu'ont suivie les musiciens de Magnitude6 semble n'avoir privilégié qu'en partie la réalité professionnelle des instrumentistes jouant d'un instrument de la famille des cuivres, cette réalité consistant davantage à être actif 
comme pigiste que comme musicien d'orchestre permanent. L'insertion professionnelle de Lalande-Markon, Jolicoeur-Côté et Latreille-Gagné dépend donc en grande partie, tel que ce fut le cas pour Demers et Champs, de leurs initiatives personnelles visant à diversifier leurs activités et à mettre sur pied ou à participer à des structures professionnelles. Sur ce dernier point, Magnitude6 agit comme un tremplin pour les jeunes professionnels qui en font partie.

\section{Exemple audio 14 : Simon Jolicoeur-Côté. Écouter.}

L'exemple des musiciens de Magnitude6 semble représentatif de la réalité de plusieurs jeunes musiciens professionnels du Québec. Ainsi, la scène musicale montréalaise a vu naître, au cours des dernières années, de nombreux ensembles constitués de musiciens en cours de formation et de jeunes professionnels jouant un rôle important dans leur professionnalisation ${ }^{11}$.

\section{BIBLIOGRAPHIE}

Becker, Howard S. ([1988]2010), Les mondes de l'art, présentation de Pierre-Michel Menger, traduit de l'anglais par Jeanne Bouniort, Paris, Flammarion.

Bourdieu, Pierre (1980), "Le capital social. Notes provisoires », Actes de la recherche en sciences sociales, vol. 31, p. 2-3.

Bureau, Marie-Christine, et Roberta Shapiro (2009), " Introduction. "Et à part ça, vous faites quoi ?" ", dans Marie-Christine Bureau, Marc Perrenoud et Roberta Shapiro (dir.), L'artiste pluriel. Démultiplier l'activité pour vivre de son art, Villeneuve-d'Ascq, Presses universitaires du Septentrion, p. 17-31.

Coulangeon, Philippe (2008), «Les transformations du marché du travail musical», dans Pierre François (dir.), La musique. Une industrie, des pratiques, Paris, La Documentation française, p. 47-63.

Liot, Françoise (2004), Le métier d'artiste, Paris, L'Harmattan.

Menger, Pierre-Michel (2009), Le travail créateur. S'accomplir dans l'incertain, Paris, Seuil/Gallimard.

Moulin, Raymonde (1992), L'artiste, l'institution et le marché, Paris, Flammarion.

Roux, Nicolas (2011), "Les "fils tendus" sur la discontinuité de l'emploi. Expérience et pratiques d'intermittents du spectacle vivant », Cahier du Lise, no 4, p. 1-23.

11 Nous retrouvons, par exemple, l'ensemble Alizé, Quasar, Pentaèdre, Forestare, Oktopus, Ex Æquo, le Duo Danaïdes et le Quintette à vent Brevà. 